\title{
Six-minute walk test protocol variations in low-resource settings - A scoping review
}

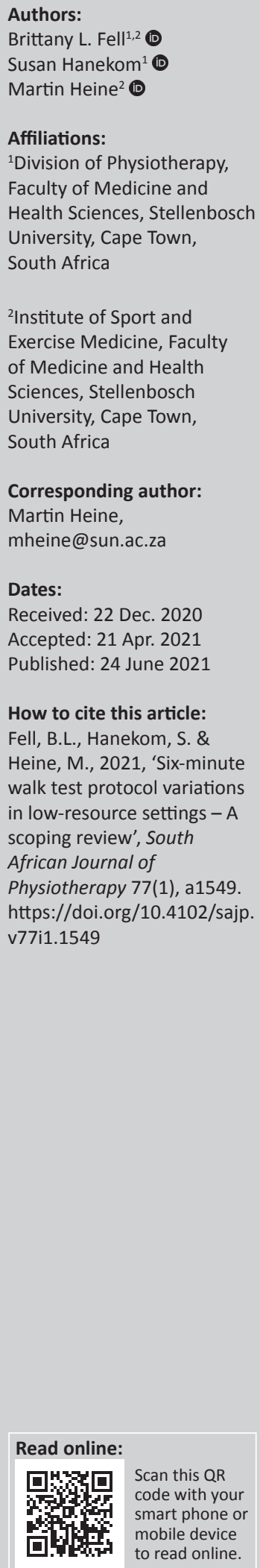

Background: The 6-min walk test (6MWT) is a validated tool, of submaximal intensity, used to objectively measure functional exercise capacity. In 2002, the American Thoracic Society (ATS) developed guidelines on standardising the implementation of the 6MWT. Despite the relative ease of conducting the $6 \mathrm{MWT}$ as per these guidelines, adaptations are implemented.

Objectives: Identify (1) what 6MWT adaptations to the ATS guidelines have been described in low-resource settings (LRS), (2) the purpose of the adapted 6MWT and (3) the reported argumentation for making these adaptations in relation to the specific context.

Methods: Five databases were searched from inception until February 2021. Studies that adapted and conducted the 6MWT in LRS were included. Data concerning the study source, participants, 6MWT: purpose, variations, outcome and rationale were extracted.

Results: A total of 24 studies were included. The majority of studies $(n=18 ; 75 \%)$ were conducted in lower-middle income countries. The most common adaptation implemented was variation to course length. Eight studies provided a rationale for adapting the 6MWT. Space constraint was the most common reason for adaptation.

Conclusion: The most common reason (space constraints) for adapting the 6MWT in LRS was addressed through adaptations in course length and/or configuration. The results of this review suggest that the value of the ATS-guided 6MWT in LRS may need to be reevaluated.

Clinical implications: Using adapted forms of the 6MWT may lead to an underestimation of a patient's abilities, misinformed discharge and developing inappropriate exercise programmes. Additionally, diverting from ATS guidelines may affect the continuity of care.

Keywords: rehabilitation; walking test; functional capacity; outcome assessment; noncommunicable diseases.

\section{Introduction}

The 6-min walk test (6MWT) is a validated tool, of submaximal intensity, used to objectively measure functional exercise capacity across numerous pathologies including patients with multiple co-morbidities (Chetta et al. 2006; Dourado et al. 2009). This field test is conducted by having a participant walk a fixed lap length over a set time period of $6 \mathrm{~min}$ (Chetta et al. 2006). In 2002, the American Thoracic Society (ATS) developed and published guidelines on how to implement the test in a standardised manner (Crapo, Enright \& Zeballos 2002). These guidelines aim to promote consistent clinical application of the test, thereby allowing comparisons, globally, across studies (Ubuane et al. 2018).

The ATS guidelines stipulate that the test is conducted indoors, on a hard-flat surface, using a $30 \mathrm{~m}$ (100 ft.) straight path (i.e. walkway or lap length), marked with cones on each end. Participants are subsequently instructed to walk as far as they can in $6 \mathrm{~min}$, and standard phrases of encouragement are used at every minute mark. The 6-min walk distance (6MWD), the primary outcome derived from the 6MWT, is calculated by adding the number of completed laps with the distance of the unfinished lap the participant was able to achieve by the end of the 6 min (Bellet et al. 2011). The 6MWD can be interpreted by comparing it to a predicted normative value, using previously established reference equations or by using it as an absolute value for comparison to a previously completed test by the same participant (Ben Saad et al. 2009). Subsequently, there are many ways one can use the 6MWD in clinical practice or research. 
Individualised exercise is the evidence-based cornerstone of any comprehensive rehabilitation programme for those with non-communicable disease, in particular those related to lifestyle (e.g. diabetes, cardiovascular disease). Improvements in physical function, through exercise, have been linked to reductions in premature mortality and morbidity (Van der Leeden et al. 2018) and clinical risk markers as well as improvements in quality of life (QOL), (Anderson \& Durstine 2019) amongst others. For instance, patients with chronic obstructive pulmonary disease (COPD) have shown improvement in QOL and a reduction in their symptoms when following a low-to-moderate intensity exercise programme. However, greater physiological responses like increased exercise capacity and decreased ventilatory demand are noted when exercise programmes are of higher intensity (Rodrigues et al. 2016). This emphasises the need for patient assessment to help guide clinicians in developing patientspecific, yet appropriate, exercise programmes.

Whilst the gold standard for the objective measurement of maximal functional capacity is cardiopulmonary exercise testing (CPET), (Ubuane et al. 2018), one can argue that this resource-heavy (i.e. equipment, training) test is often not feasible or necessary. Conversely, the 6MWT is often recommended as the field test of choice in day-to-day clinical practice. Reasons for this include its applicability in various populations, physiological systems and settings. Additionally, the test is easy to perform, not time-consuming, does not require additional equipment or special skills and is also more representative of the exertion of everyday activities (Bellet et al. 2011; Chetta et al. 2006; Ubuane et al. 2018).

However, despite the relative ease of conducting the 6MWT in a standardised way, particularly in comparison to CPET, adaptations to the 'ATS-guided $6 \mathrm{MWT}^{\prime}$ are being implemented (Sciurba et al. 2003). Typically, these adaptations may include changes to walkway distance and configuration, the inclusion of a practice run or test instructions, and encouragement, etc. Subsequently, the 6MWD may be influenced by these adaptations, (Barnett et al. 2016; Casillas et al. 2013), for instance, changes in gait speed, pacing strategy adopted and number of turns made to complete the test (Barnett et al. 2016; Ng et al. 2013). For example, Beekman et al. (2013) showed in a study involving patients with COPD that reducing pathway distance from $30 \mathrm{~m}$ to $10 \mathrm{~m}$ resulted in an average $49.5 \mathrm{~m}$ shorter distance. The difference in 6MWD between the two pathway distances was attributed to the increased number of turns on the $10 \mathrm{~m}$ pathway, and subsequently less time spent at optimal walking pace (Beekman et al. 2013). This discrepancy in 6MWD, in this case because of a difference in pathway length, may have clinical implications. Firstly, if one would compare the $10 \mathrm{~m}$ results to establish reference equations developed using ATS guidelines, it is likely that the patient's functional abilities will be markedly underestimated. Secondly, the shorter distance achieved by the participants on the $10 \mathrm{~m}$ pathway $(49.5 \mathrm{~m})$ is larger than the reported minimum clinically important difference (MCID) of $35 \mathrm{~m}$ as reported by Puhan and colleagues, as well as the $30 \mathrm{~m}$
MCID reported by Polkey and colleagues (Polkey et al. 2013; Puhan et al. 2008). Thirdly, using data based on different $6 \mathrm{MWT}$ protocols interchangeably could lead to either premature or delayed discharge of patients as well as the prescription of an inappropriate exercise programme, amongst others.

In cases of a misinformed discharge (an extended hospital admission or early discharge), this may have a compounding effect, specifically in already resource-constrained or overburdened healthcare environments. Collectively, these examples highlight the importance of using standardised testing protocols, particularly in settings of low resources, (Van Zyl et al. 2021) where the gold standard for CPET is often unavailable and physical resources (e.g. space) may be limited.

The objective of our scoping review is therefore to identify: (1) what 6MWT adaptations to the ATS guidelines have been described in low-resource settings (LRS), (2) the purpose of the adapted 6MWT and (3) the reported argumentation for making these adaptations in relation to the specific context.

\section{Methods}

Our scoping review was conducted in accordance with the framework provided by Arksey and O'Malley (Arksey \& O'Malley 2005) and reported according to the PRISMA guidelines including scoping review extensions (Tricco et al. 2018) (see online Supplement 1).

\section{Data sources and search strategy}

Five bibliographical databases were accessed through the Stellenbosch University Library: Cochrane Library, EBSCOhost (AfricaWide, Cumulative Index to Nursing and Allied Health Literature [CINAHL], Medline), PubMed, Scopus and Web of Science. An initial search was conducted (BF) from inception to 31 October 2019. Subsequently, the search was updated to include records up to 14 February 2021. Search terms included variations of the following main search terms: 6MWT, low-resource setting and developing countries (see online Supplement 2).

\section{Study selection}

After the completion of the searches, removal of duplicates, titles and abstracts were independently screened by two reviewers (BF and $\mathrm{MH})$. Any disagreements were discussed by the reviewers and a third reviewer was consulted $(\mathrm{SH})$ in the case of irreconcilable disagreements. An identical procedure was followed for full text screening to determine final full text inclusions. All original research study designs that were available in English were considered. Included studies must have reported on and used an adapted or modified version of the $6 \mathrm{MWT}$ and conducted the test within the context of low-resource setting (Van Zyl et al. 2021). In the absence of a clear definition for LRS, within the context of an upper middle income country (UMIC) or high income country (HIC), studies were included based on the language (e.g. rural) used by the study in conjunction with online resources 
(detail on the location or context of a specific clinic) and purposeful discussion amongst the authors. All ages were considered for our review. After becoming familiar with the sources, decisions were made to exclude case studies and case series and any studies that performed the $6 \mathrm{MWT}$ according to ATS guidelines or did not report on the methods used. Despite searching online resources from inception, articles published before 2002 (year of published ATS guidelines) were excluded.

\section{Data extraction and synthesis}

A data extraction form was developed, and revised as necessary, to extract relevant data from the included full-text articles and captured under the following headings: source, participants, reported 6MWT purpose, adaptations, outcomes and rationale. In case the rationale for making adaptations was unclear, corresponding authors were contacted. Data were extracted by the primary reviewer $\mathrm{BF}$ and verified independently by a second reviewer $(\mathrm{MH})$. In the event of group data (e.g. male and female, or intervention and control group) being reported separately, an aggregated 6MWD mean and standard deviations were calculated for each study, using the formula provided in the Cochrane handbook; excluded data were reported separately for diseased groups with healthy controls (Higgins et al. 2019). In case of longitudinal studies, only baseline data were extracted.

\section{Concepts and context}

\section{Low-resource setting}

In line with previous research, a low-resource setting was defined as a low-income country (LIC) or lower-middle income country (LMIC) as per the World Bank Criteria, (World Bank 2018), or in the event of an UMIC or HIC, an explicit statement indicating a LRS (e.g. rural, minority populations, poverty) was required (Heine et al. 2019).

\section{Six-minute walk test adaptations}

The test was considered adapted when it deviated from the 2002 ATS guidelines for the 6MWT, (Enright 2003) and grouped in the following categories:

- distance; shorter or longer than $30 \mathrm{~m}$ (100 ft.)

- configuration, that is, not a straight pathway

- venue; not indoors

- instructions or encouragement were not as provided by the ATS guidelines

- 'other' (e.g. treadmill).

\section{Ethical considerations}

This article followed all ethical standards for research without direct contact with human or animal subjects.

\section{Results}

The PRISMA flow chart is shown in Figure 1. Of the 564 records identified across the five databases, 724 remained after removing duplicates. After the initial title and abstract

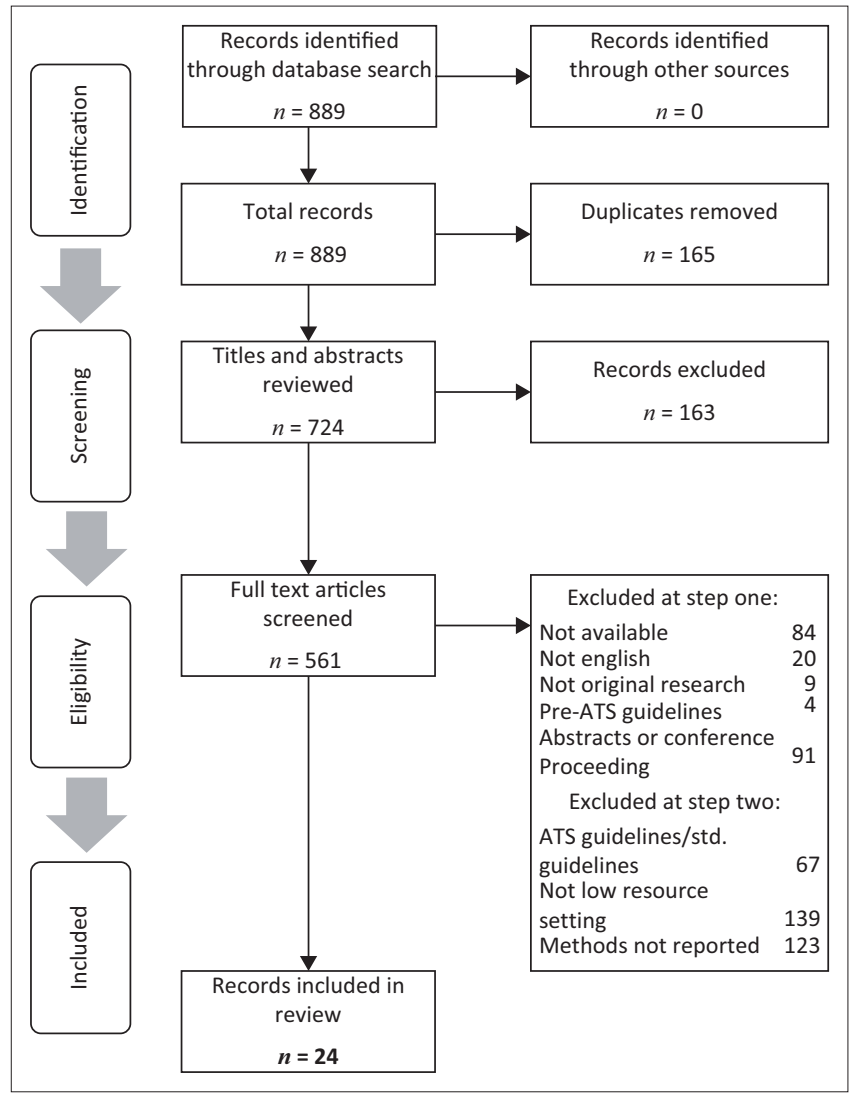

FIGURE 1: Preferred Reporting Items for Systematic Reviews and Meta-Analyses (PRISMA) flow chart of data synthesis.

screening, 342 articles were identified for full text screening. Available full texts were screened for eligibility, resulting in the exclusion of 537 articles. Reasons for exclusion are provided (Figure 1). A total of 24 studies were included in our review.

An overview of all included studies $(n=24)$ is provided (Table 1). The earliest study included in our review was published in 2011, whilst remaining studies were published between 2012 and 2020. Various study designs were included, with randomised controlled trials $(n=10 ; 42 \%)$ being the most frequent. The most common diagnoses in which an adapted 6MWT was reported include respiratory disease $(n=8 ; 33 \%)$ and cardiovascular disease $(n=4 ; 17 \%)$. Studies were conducted predominantly in LMICs $(n=18 ; 75 \%)$, followed by four studies (17\%) conducted in LIC (Benin, Malawi and Uganda), whilst two (8\%) studies were conducted in a low-resource context of either an UMIC (Brazil) or HIC (Australia).

\section{Six-minute walk test purpose}

The majority of included studies (58\%) reported using the $6 \mathrm{MWT}$ as a measure of submaximal exercise or functional capacity to determine the effect or effectiveness of an intervention in various pathologies. Other purposes of the $6 \mathrm{MWT}$ included validation of the 6MWT against other measures, (Nusdwinuringtyas et al. 2014; Sogbossi, Thonnard \& Batcho 2014), correlation of $6 \mathrm{MWT}$ with 2-min walk test, 


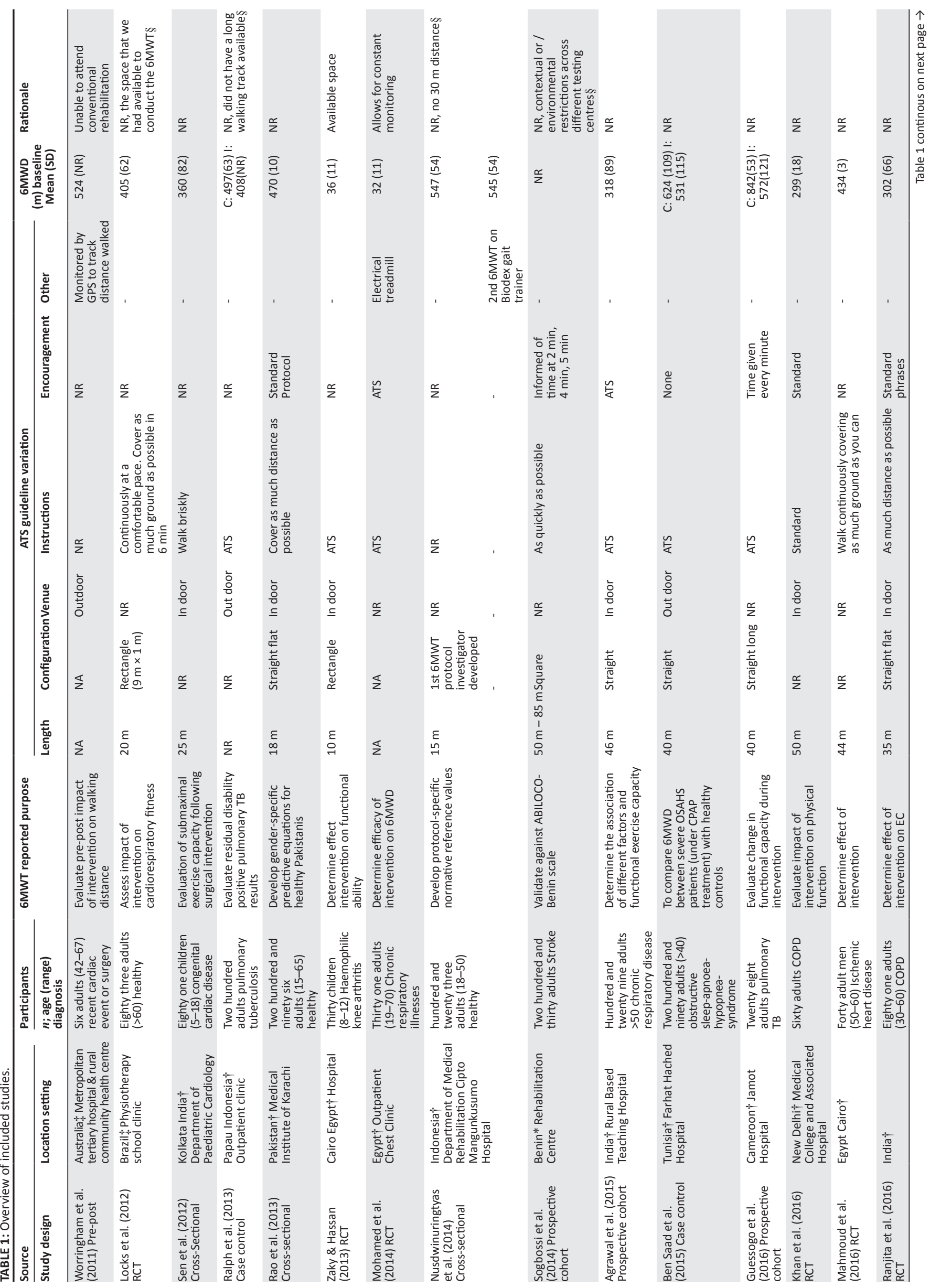




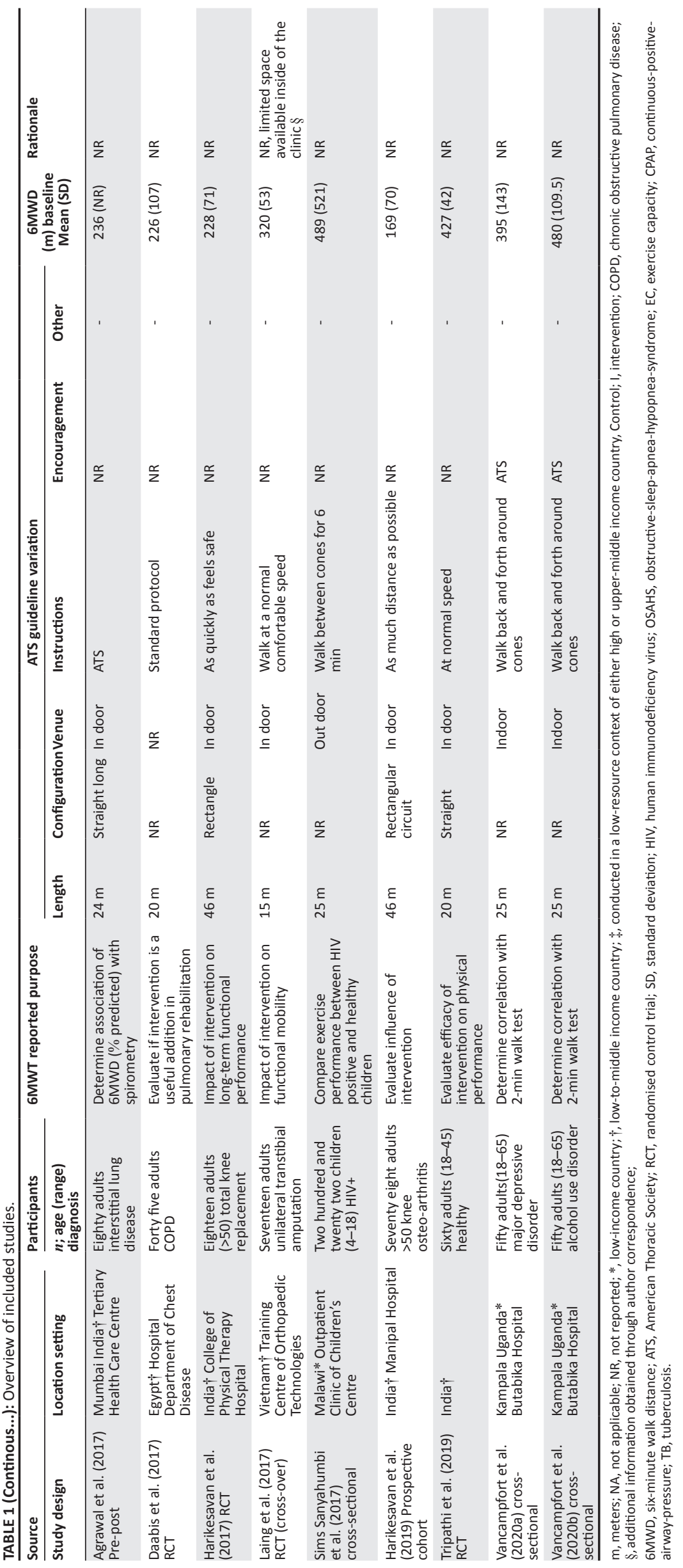


(Vancampfort et al. 2020a, 2020b), developing normative values, (Nusdwinuringtyas et al. 2014; Rao et al. 2013) or as a descriptive variable in association with lung spirometry or QOL.

\section{Six-minute walk test adaptations}

An overview of the adaptations made by the included studies is provided (Figure 2). The most common adaptation made to the ATS guidelines was that of course length $(n=21 ; 88 \%)$ ranging between $10 \mathrm{~m}$ and $85 \mathrm{~m}$ per lap. Of studies that adapted the course length, 12 (50\%) reported a shorter pathway and $9(38 \%)$ reported a pathway distance longer than $30 \mathrm{~m}$. Twelve studies reported using a different configuration than straight, including using a rectangular (Harikesavan, Chakravarty \& Maiya 2019; Harikesavan et al. 2017; Locks et al. 2012; Zaky \& Hassan 2013) or square (Sogbossi et al. 2014) layout. One study reported using a 6MWT protocol developed by the study's investigator (Nusdwinuringtyas et al. 2014). Four studies (17\%) specifically reported conducting the 6MWT outdoors, 13 (54\%) studies conducted the test indoors, as per ATS guidelines, whilst the remaining studies $(n=7 ; 29 \%)$ did not report detail on the test venue.

Instructions as per the ATS Guidelines were used in seven (29\%) of the studies, whilst $13(54 \%)$ studies reported instructions that were modified to include effort (e.g. walk briskly) or objective (e.g. cover as much distance as possible) (Rao et al. 2013).

The use of encouragement was generally not well reported, and standardised phrases were used $(n=7 ; 29 \%)$, whilst

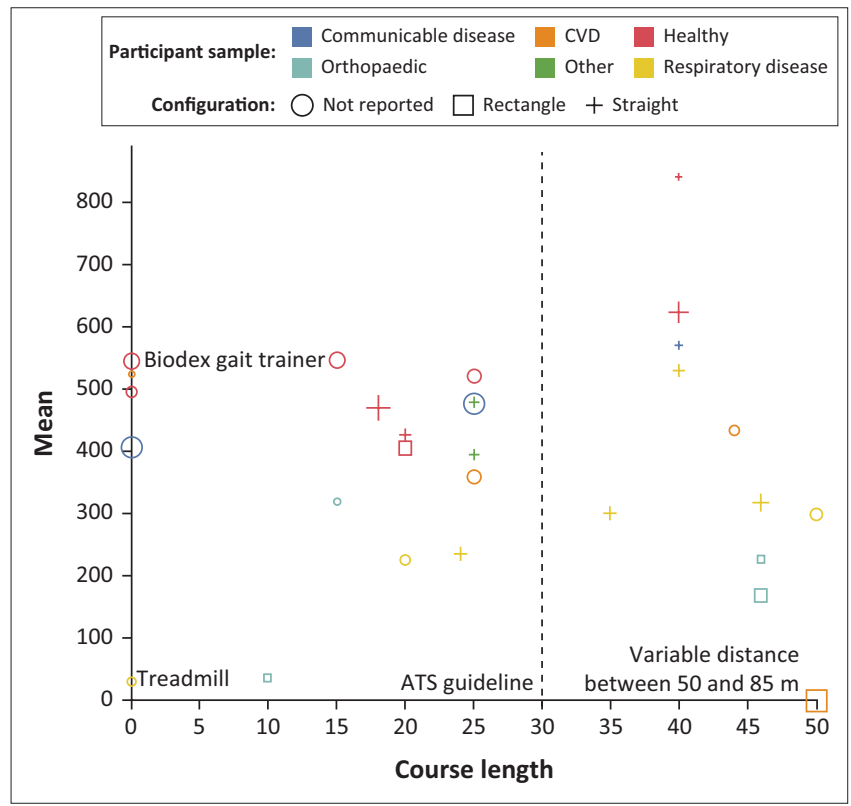

CVD, cardiovascular disease; ATS, American Thoracic Society.

Rationale for making adaptations.

FIGURE 2: A graphical summary of the six-minute walk test variations for included studies $(n=24)$. adapted $(n=2 ; 8 \%)$ encouragements included giving time at non-standardised time intervals (Sogbossi et al. 2014). Some studies refrained from using any encouragement during the test. Three studies included in this review reported 'other' adaptations which could all be linked to the use of technology. Nusdwinuringtyas et al. (2014) compared a conventional 6MWT (although $15 \mathrm{~m}$ course length) with one performed on a Biodex ${ }^{\circledR}$ gait trainer, reporting a non-significant mean difference of $2.73 \mathrm{~m}$. Similarly, Mohamed et al. (2014) used a treadmill (controlled by the patient) to perform their test. Finally, Worringham et al. (2014) reported using global positioning system (GPS) monitoring to remotely track the distances walked by patients in a natural environment over the course of $6 \mathrm{~min}$.

Eight of the included studies, five of which through author correspondence, provided a specific rationale for using an adapted 6MWT relative to a conventional ATS guideline version. Space constraint, as a result of environmental conditions, was the most common rationale for adapting the 6MWT (Laing et al. 2017; Locks et al. 2012; Nusdwinuringtyas et al. 2014; Ralph et al. 2013; Sogbossi et al. 2014; Zaky \& Hassan 2013). Worringham, Rojek and Stewart (2011) reported conducting the test off-site for participants unable to attend standard rehabilitation sessions, whilst Mohammed et al. (2014) reported that using a treadmill (stationary test) allows for continuous monitoring of patient parameters.

\section{Discussion}

To the best of our knowledge, this is the first study to explore adaptations to the ATS guidelines for conducting a 6MWT, in conjunction with the rationale for making these changes, specifically in LRS. Globally, LRS are seeing a significant, albeit slow, shift in disease burden from communicable disease towards non-communicable diseases (Murray et al. 2020). Because of the nature of the test, its applicability to assist in clinical decision-making, and its association with important outcomes like hospitalisation and mortality, there are compelling arguments for including the 6MWT as a key measure across the continuum of care. Standardisation in conducting the test is paramount from several perspectives including: the construct validity of the test as a measure of functional capacity, the within-patient homogeneity moving through various tiers of the healthcare system and ability to synthesise research outputs across settings for guideline development. Twenty-four studies were identified that predominantly reported variations in course length and configuration. In general, the methods used for conducting the 6MWT were poorly reported. Our review therefore assists in understanding the adaptations to the 6MWT ATS guidelines, drafted in 2002, made in LRS to inform the academic and clinical landscape and potentially consider the need for reconsidering these guidelines in the light of space constraints or other resource limitations (e.g. rurality).

In looking at the specific adaptations, space limitations specifically, were most commonly reported as a reason for shortening the course length. Unexpectedly, yet without 
clear reasons provided, almost half of studies included in this review reported using a longer (as opposed to shorter) course length, although, such a longer course length was often combined with a course reconfiguration (e.g. $80 \mathrm{~m}$, square). Arguably, in studies where course length was increased and configuration altered, the course lay-out was adjusted pragmatically to adjust for the available space most likely to be interrupted. For instance, a short $10 \mathrm{~m}$ and straight course can be hypothesised to be more interruptive in that specific hallway compared to a rectangular $80 \mathrm{~m}$ 'system of hallways'. The alternative to changing course length or configuration is to move the test from indoor to outdoor, as some studies in this review opted for (Ben Saad et al. 2015; Ralph et al. 2013; Sanyahumbi et al. 2017). Under reasonable weather conditions, no significant difference in 6MWD between indoor and outdoor 6MWT test settings has been reported, therefore opening up a possibly more feasible avenue to retain the standard ATS course configuration (Brooks et al. 2003).

The study samples in which the 6MWT was used were diverse, ranging from non-communicable disease to communicable disease, as well as mental health disorders, amongst others. The wide range of populations, beyond 'cardiorespiratory' indications, illustrates the wide use and value of the 6MWT for research and clinical practice. In most cases, the purpose for including the 6MWT was either as a measure of functional capacity to be associated with a second outcome measure (e.g. 6MWT vs. lung function, or $6 \mathrm{MWT}$ vs. the 2-min walk test), to allow for comparison between study samples, or as an outcome measure in studies of efficacy. The impact of diverting from the ATS standards can be considered different depending on the purpose for which the $6 \mathrm{MWT}$ was used, in particular in conjunction with reference values based on different configurations. In our review, two studies used (inadequate) reference equations for the interpretation of findings. Whilst, because pre-existing reference equations either over or underestimated the abilities of their patient population (Indonesian and Pakistani), two studies developed reference equations specific to their population anthropometrics. Additionally, one study in an Indian patient population found a significant association between the 6MWT and pulmonary hypertension when using the Indian reference equation. When the same analysis was completed using a different reference equation, correlation could not be determined. In an academic context, diverting from ATS guidelines might be less problematic, in particular in the context of pre-to-post testing when evaluating within patient or group changes over time. However, in clinical settings, this may lead to the lack of continuity as referred to earlier.

Reasons for adaptation were reported inconsistently, yet mostly pertained to space constraints. Interestingly, space constraints may not be endemic to LRS per se (albeit the implications in LRS may be more pronounced). For instance, Thaweewannakij et al. (2013) reported using a $6 \mathrm{~m} \times 4 \mathrm{~m}$ rectangular walkway because of the absence of a large enough walkway in communities (Thailand, Upper-Middle Income Country), where the tests were conducted (Thaweewannakij et al. 2013). Furthermore, the notion of 'LRS' is more encompassing than a lack of space (Van Zyl et al. 2021). Barriers in service delivery, such as the logistics in accessing care, also need to be considered (Heller et al. 2019). In this light, the GPS-based 6MWT, to be used in a reallife environment, (Worringham et al. 2011) is a particularly interesting technological advancement, when moving towards telehealth and access to rural populations.

\section{Limitations}

Our review has some limitations. Firstly, only studies that were published in English were considered. Secondly, the methods used for conducting the 6MWT, if reported, could often only be derived at full-text review stage. For many studies at full-text review, it was unclear as to the exact methods used. Given that some of the included studies in our review referred to the ATS guidelines as the 6MWT protocol, still reported adaptations thereof may indicate that adaptations were missed in those studies referring to the ATS guidelines with no further detail reported. Thirdly, additional adaptations could have been derived from studies conducted in high-resource settings not included in this review. Fourthly, it is also worth noting one specific adaptation to the 6MWT that was not considered in the scope of our review. The 2-min walk test or other adaptations to the duration of the test and its potential practical implications (e.g. time savings) were not studied. Finally, the impact of the adaptations on the primary outcome (6MWD) could not be derived from the data because of the underlying heterogeneity. Surprisingly, few studies reported validating their adapted 6MWT protocols against the gold standard.

\section{Conclusion}

Space constraint is the most common reported reason for diverting from the ATS guidelines when using the 6MWT in LRS. Adaptations were made to the course length, or configuration to address these space limitations, often in conjunction. The ATS guidelines may need to be revisited, or context-specific norm values need to be developed, in order for the $6 \mathrm{MWT}$ to be more conducive to LRS (or other settings with space limitations).

\section{Acknowledgements Competing interests}

The authors declare that they have no financial or personal relationships that may have inappropriately influenced them in writing this article.

\section{Authors' contributions}

All authors contributed to the manuscript. B.F. conceptualised our review, conducted the review process and wrote the 
initial draft. In addition to the conceptualisation of this review, S.H. and M.H. provided ongoing supervision, project management and reviewed and edited the manuscript draft.

\section{Funding information}

This research received no specific grant from any funding agency in the public, commercial or not-for-profit sector.

\section{Data availability}

The authors confirm that the data supporting the findings of this study are available within the article.

\section{Disclaimer}

The views and opinions expressed in this article are those of the authors and do not necessarily reflect the official policy or position of any affiliated agency of the authors.

\section{References}

Agrawal, S.R., Joshi, R. \& Jain, A., 2015, 'Correlation of severity of chronic obstructive pulmonary disease with health-related quality of life and six-minute walk test in a rural hospital of central India', Lung India 32(3), 233. https://doi. org/10.4103/0970-2113.156231

Anderson, E. \& Durstine, J.L., 2019, 'Physical activity, exercise, and chronic diseases: A brief review', Sports Medicine and Health Science 1(1), 3-10. https://doi. org/10.1016/j.smhs.2019.08.006

Arksey, H. \& O'Malley, L., 2005, 'Scoping studies: Towards a methodological framework', International Journal of Social Research Methodology: Theory and Practice 8(1), 19-32. https://doi.org/10.1080/1364557032000119616

Barnett, C.T., Bisele, M., Jackman, J.S., Rayne, T., Moore, N.C., Spalding, J.L. et al 2016, 'Manipulating walking path configuration influences gait variability and sixminute walk test outcomes in older and younger adults', Gait and Posture 44, 221-226. https://doi.org/10.1016/j.gaitpost.2015.12.022

Beekman, E., Mesters, I., Hendriks, E.J.M., Klaasen, M.P.M., Gosselink, R., Van Schayck, O.C.P. et al., 2013, 'Course length of 30 metres versus 10 metres has a significant influence on six-minute walk distance in patients with COPD: An experimental crossover study', Journal of Physiotherapy 59(3), 169-176. https://doi org/10.1016/S1836-9553(13)70181-4

Bellet, R.N., Francis, R.L., Jacob, J.S., Bartlett, H.J., Adams, L., Morris, N.R. et al., 2011 'Repeated six-minute walk tests for outcome measurement and exercise prescription in outpatient cardiac rehabilitation: A longitudinal study', Archives of Physical Medicine and Rehabilitation 92(9), 1388-1394. https://doi.org/10.1016/j. apmr.2011.04.014

Ben Saad, H., Prefaut, C., Tabka, Z., Hadj Mtir, A., Chemit, M., Hassaoune, R. et al., 2009 , '6-minute walk distance in healthy North Africans older than 40 years: Influence of parity', Respiratory Medicine 103(1), 74-84. https://doi. org/10.1016/j.rmed.2008.07.023

Ben Saad, H., Hassen, I.B., Ghannouchi, I., Latiri, I., Rouatbi, S., Escourrou, P. et al., 2015, '6-min walk-test data in severe obstructive-sleep-apnea-hypopneasyndrome (OSAHS) under continuous-positive-airway-pressure (CPAP) treatment', Respiratory Medicine 109(5), 642-655. https://doi.org/10.1016/j. rmed.2015.03.001

Brooks, D., Solway, S., Weinacht, K., Wang, D. \& Thomas, S., 2003, 'Comparison between an indoor and an outdoor 6-minute walk test among individuals with chronic obstructive pulmonary disease', Archives of Physical Medicine and Rehabilitation 84(6), 873-876. https://doi.org/10.1016/50003M993(03)00011-X

Casillas, J.M., Hannequin, A., Besson, D., Benaim, S., Krawcow, C., Laurent, Y. et al. 2013, 'Walking tests during the exercise training: Specific use for the cardiac rehabilitation', Annals of Physical and Rehabilitation Medicine 56(7-8), 561-575. rehabilitation', Annals of Physical and Rehabilite
https://doi.org/10.1016/j.rehab.2013.09.003

Chetta, A., Zanini, A., Pisi, G., Aiello, M., Tzani, P., Neri, M. et al., 2006, 'Reference values for the 6-min walk test in healthy subjects $20-50$ years old', Respiratory Medicine 100(9), 1573-1578. https://doi.org/10.1016/j.rmed.2006.01.001

Crapo, R., Enright, P. \& Zeballos, J., 2002, 'American thoracic society ATS statement: Guidelines for the six-minute walk test', American Journal of Respiratory and Critical Care Medicine 166(1), 111-117. https://doi.org/10.1164/ ajrccm.166.1.at1102

Daabis, R., Hassan, M. \& Zidan, M., 2017, 'Endurance and strength training in pulmonary rehabilitation for COPD patients', Egyptian Journal of Chest Diseases and Tuberculosis 66(2), 231-236. https://doi.org/10.1016/j. ejcdt.2016.07.003
Dourado, V.Z., Tanni, S.E., Antunes, L.C.O., Paiva, S.A.R., Campana, A.O., Renno, A.C.M. et al., 2009, 'Effect of three exercise programs on patients with chronic obstructive pulmonary disease', Brazilian Journal of Medical and Biological Research 42(3), 263-271. https://doi.org/10.1590/S0100-879X2009000300007

Enright, P.L., 2003, 'The six-minute walk test', Respiratory Care 48(8), 783-785.

Guessogo, W.R., Mandengue, S.H., Ndemba, P.B.A., Medjo, U.O., Minye, E.E., Ahmaidi S., et al., 2016, 'Physical and functional follow-up of tuberculosis patients in initia intensive phase of treatment in Cameroon using the 6-min walk test' Journal of Exercise Rehabilitation 12(4), 333-339. https://doi.org/0.12965/jer.1632620.310

Harikesavan, K., Chakravarty, R.D.D. \& Maiya, A.G., 2019, 'Influence of early mobilization program on pain, self-reported and performance based functional measures following total knee replacement', Journal of Clinical Orthopaedics and Trauma 10(2), 340-344. https://doi.org/10.1016/j.jcot.2018.04.017

Harikesavan, K., Chakravarty, R.D.D., Maiya, A.G., Hegde, S.P. \& Shivanna, S.Y. 2017, 'Hip abductor strengthening improves physical function following total knee replacement: One-year follow-up of a randomized pilot study', The Open Rheumatology Journal 11(1), 30-42. https://doi.org/10.2174/187431290171
1010030

Heine, M., Lupton-Smith, A., Pakosh, M., Grace, S.L., Derman, W. \& Hanekom, S.D. 2019, 'Exercise-based rehabilitation for major non-communicable diseases in lowresource settings: A scoping review', BMJ Global Health 4(6), 1-10. https://doi. resource settings: A scoping review,
org/10.1136/bmjgh-2019-001833

Heller, D.J., Kumar, A., Kishore, S.P., Horowitz, C.R., Joshi, R. \& Vedanthan, R., 2019, 'Assessment of barriers and facilitators to the delivery of care for noncommunicable diseases by nonphysician health workers in low- and
middle-income countries: A systematic review and qualitative analysis', JAMA Network Open 2(12), e1916545. https://doi.org/10.1001/jamanetworkopen. Network Open
2019.16545

Higgins, J.P., Thomas, J., Chandler, J., Cumpston, M., Li, T., Page, M.J. \& Welch, V.A. (eds.)., 2019, Cochrane handbook for systematic reviews of interventions, John Wiley \& Sons, Chichester, United Kindom.

Khan, N.A., Kumar, N. \& Daga, M.K., 2016, 'Effect of dietary supplementation on body composition, pulmonary function and health-related quality of life in patients with stable COPD', Tanaffos 15(4), 225-235.

Laing, S., Lythgo, N., Lavranos, J. \& Lee, P.V.S., 2017, 'Transtibial prosthetic socket shape in a developing country: A study to compare initial outcomes in pressure cast hydrostatic and patella tendon bearing designs', Gait \& Posture 58, 363-368. https://doi.org/10.1016/j.gaitpost.2017.08.017

Locks, R.R., Costa, T.C., Koppe, S., Yamaguti, A.M., Garcia, M.C. \& Gomes, A.R.S., 2012, 'Effects of strength and flexibility training on functional performance of healthy older people', Brazilian Journal of Physical Therapy 16(3), 184-190. https://doi. org/10.1590/S1413-35552012000300003

Mahmoud, H.H., Mohamed, N.G., Mohamed, A.R. \& Ewas E.B., 2016, 'Adipokines response to continuous versus interval aerobic training in ischaemic heart disease response to continuous versus interval aerobic training in ischaemic
patients', International Journal of PharmTech Research 9(10), 53-9.

Mohamed, A.R. \& Shaban, M.M., 2014, 'Role of laser acupuncture in chronic respiratory diseases', The Egyptian Journal of Chest Diseases and Tuberculosis 63(4), 1065-1070. https://doi.org/10.1016/j.ejcdt.2014.06.012

Murray, C.J., Abbafati, C., Abbas, K.M. \& Abbasi, M., 2020, 'Five insights from the global burden of disease study 2019', 396(10258), 1135-1159. https://doi. org/10.1016/S0140-6736(20)31404-5

Ng, S.S., Yu, P.C., To, F.P., Chung, J.S. \& Cheung, T.H., 2013, 'Effect of walkway length and turning direction on the distance covered in the 6-minute walk test amon adults over 50 years of age: A cross-sectional study', Physiotherapy 99(1), 63-70. https://doi.org/10.1016/j.physio.2011.11.005

Nusdwinuringtyas, N., Widjajalaksmi, W., Yunus, F. \& Alwi, I., 2014, 'Reference equation for prediction of a total distance during six-minute walk test using Indonesian anthropometrics', Acta Medica Indonesiana 46(2), 90-96.

Polkey, M.I., Spruit, M.A., Edwards, L.D., Watkins, M.L., Pinto-Plata, V., Vestbo, J. et al., 2013, 'Six-minute-walk test in chronic obstructive pulmonary disease: Minimal 2013, 'Six-minute-walk test in chronic obstructive pulmonary disease: Minimal
clinically important difference for death or hospitalization', American Journal of clinically important difference for death or hospitalization', American Journal of Respiratory and Critica
rccm.201209-15960C

Puhan, M.A., Mador, M.J., Held, U., Goldstein, R., Guyatt, G.H. \& Schunemann, H.J., 2008 , 'Interpretation of treatment changes in 6 -minute walk distance in patients with COPD', European Respiratory Journal 32(3), 637-643. https://doi. with COPD', European Respiratory
org/10.1183/09031936.00140507

Ralph, A.P., Kenangalem, E., Waramori, G., Pontororing, G.J., Sandjaja, Tjitra, E. et al., 2013, 'High morbidity during treatment and residual pulmonary disability in pulmonary tuberculosis: Under-recognised phenomena', PLoS One 8(11), 1-11. https://doi.org/10.1371/journal.pone.0080302

Ranjita, R., Hankey, A., Nagendra, H.R. \& Mohanty, S., 2016, 'Yoga-based pulmonary rehabilitation for the management of dyspnea in coal miners with chronic obstructive pulmonary disease: A randomized controlled trial', Journal of Ayurveda and Integrative Medicine 7(3), 158-166. https://doi.org/10.1016/j.jaim.2015.12.001

Rao, N. A., Irfan, M., Haque, A. S., Zubairi, A. B. S. \& Awan, S., 2013, 'Six-minute walk test performance in healthy adult Pakistani volunteers.', Journal of the College of Physicians and Surgeons - Pakistan: JCPSP 23(10), 720-725.

Rodrigues, A., Martino, M.D., Nellessen, A.G., Hernandes, N.A., Neder, J.A. \& Pitta, F., 2016, 'Is the six-minute walk test a useful tool to prescribe high-intensity exercise in patients with chronic obstructive pulmonary disease?', Heart and Lung: Journal of Acute and Critical Care 45(6), 550-556. https://doi.org/10.1016/j. hrtlng.2016.08.005

Sanyahumbi, A.E.S., Hosseinipour, M.C., Guffey, D., Hoffman, I., Kazembe, P., McCrary, M. et al., 2017, 'HIV-infected children in Malawi have decreased performance on the 6 -minute walk test with preserved cardiac mechanics regardless of antiretroviral treatment status', Pediatric Infectious Disease Journal 36(7), 659-664. https://doi.org/10.1097//NF.0000000000001540 
Sciurba, F., Criner, G.J., Lee, S.M., Mohsenifar, Z., Shade, D., Slivka, W. et al., 2003 'Six-minute walk distance in chronic obstructive pulmonary disease', American Journal of Respiratory and Critical Care Medicine 167(11), 1522-1527. https://doi. Journal of Respiratory and Critical Car
$\mathrm{org} / 10.1164 /$ rccm.200203-1660C

Sen, S., Bandyopadhyay, B., Eriksson, P. \& Chattopadhyay, A., 2012, 'Functional Capacity Following Univentricular Repair-Midterm Outcome', Congenital Heart Disease 7(5), 423-432. http://doi.wiley.com/10.1111/j.1747-0803.2012.00640.x

Sogbossi, E.S., Thonnard, J.L. \& Batcho, C.S., 2014, 'Assessing locomotion ability in West African stroke patients: Validation of ABILOCO-benin scale', Archives of Physical Medicine and Rehabilitation 95(8), 1470-1476. https://doi.org/10.1016/j. apmr.2014.03.002

Thaweewannakij, T., Wilaichit, S., Chuchot, R., Yuenyong, Y., Saengsuwan, J., Siritaratiwat, W. et al., 2013, 'Reference values of physical performance in Tha elderly people who are functioning well and dwelling in the community', Physical Therapy 93(10), 1312-1320. https://doi.org/10.2522/ptj.20120411

Tricco, A.C., Lillie, E., Zarin, W., O’Brien, K.K., Colquhoun, H., Levac, D. et al., 2018 'PRISMA extension for scoping reviews (PRISMA-SCR): Checklist and explanation', Annals of Internal Medicine 169(7), 467-473. https://doi.org/10.7326/M18-0850

Tripathi, R.K., Dethe, P.D., Bhojne, S.K., Raut, A.A. \& Rege, N.N., 2019, 'A prospective, randomized, placebo-controlled, double-blind comparative pilot study to evaluate the efficacy of Chlorophytum borivilianum on physical performance', Indian Journal of Pharmacology 51(3), 150-156. https://doi.org/10.4103/ijp. IJP_115_18

Ubuane, P.O., Animasahun, B.A., Ajiboye, O.A., Kayode-Awe, M.O., Ajayi, O.A. \& Njokanma, F.O., 2018, 'The historical evolution of the six-minute walk test as a measure of functional exercise capacity: A narrative review', Journal of Xiangya Medicine 3(3), 40. https://doi.org/10.21037/jxym.2018.11.01
Vancampfort, D., Basangwa, D., Kimbowa, S., Firth, J., Schuch, F., Van Damme, T. et al., 2020a, 'Test-retest reliability, validity, and correlates of the 2-min walk test in 2020 a, 'Test-retest reliability, validity, and correlates of the 2-min walk test in
outpatients with depression', Physiotherapy Research International 25(2), e1821. https://doi.org/10.1002/pri.1821

Vancampfort, D., Kimbowa, S., Basangwa, D., Hallgren, M., Damme, T.V., Rosenbaum, S. et al., 2020b 'Test-retest reliability, concurrent validity and correlates of the two-minute walk test in outpatients with alcohol use disorder', Alcohol $90,74-79$. https://doi.org/10.1016/j.alcohol.2020.12.001

Van der Leeden, M., Stuiver, M.M., Huijsmans, R., Geleijn, E., De Rooij, M. \& Dekker, J., 2018, 'Structured clinical reasoning for exercise prescription in patients with comorbidity', Disability and Rehabilitation 42(10), 1474-1479. https://doi.org/ 10.1080/09638288.2018.1527953

Van Zyl, C., Badenhorst, M., Hanekom, S. \& Heine, M., 2021, 'Unravelling the concept of "low-resource settings" in the context of rehabilitation: A systematic scopin review with qualitative content analysis', BMJ Global Health 0:e005190. https:// doi.org/10.1136/bmjgh-2021-005190

World Bank, 2018, The World Bank, viewed 11 March 2020, from https://datahelpdesk worldbank.org/knowledgebase/articles/906519-world-bank-country-andlending-groups.

Worringham, C., Rojek, A. \& Stewart, I., 2011, 'Development and feasibility of a smartphone, ECG and GPS-based system for remotely monitoring exercise in cardiac rehabilitation', PLoS One 6(2), e14669. https://doi.org/10.1371/journal. pone.0014669

Zaky, L.A. \& Hassan, W.F., 2013, 'Effect of partial weight bearing program on functional ability and quadriceps muscle performance in hemophilic knee arthritis', Egyptian Journal of Medical Human Genetics 14(4), 413-418. https://doi.org/10.1016/j. ejmhg.2013.02.002 